\title{
Subject awareness in differential classical eyelid conditioning
}

\author{
WILLIAM A. BENISH and DAVID A. GRANT \\ University of Wisconsin, Madison, Wisconsin 53706
}

\begin{abstract}
Four treatment groups were employed in a differential classical eyelid conditioning study. The discrimination required of the four groups was of varying complexity and was sufficiently difficult to produce a substantial number of subjects who were not aware of the reinforcement contingencies. Awareness was assessed by a postexperimental questionnaire. The findings were consistent with the generalization that awareness is a necessary but not sufficient condition for differential responding. Moreover, it was found that the probability of a subject's learning to discriminate, conditional upon his being aware, was independent of treatment condition.
\end{abstract}

Cognitive variables have been shown to be significant correlates of performance in differential classical eyelid conditioning (Grant, 1973; Ross, 1971). Of particular interest has been the relationship between a subject's discrimination performance and his ability to report the reinforcement contingencies. Grant (1973) examined this relationship and concluded that awareness at some early stage of the experiment may be necessary for differential responding to occur, but that awareness is not a sufficient condition for differential responding.

Grant (1973) also pointed to a difficulty in exploring this issue, in that assessments of awareness based on postexperimental questioning typically find that almost every subject is able to report accurately the relationship between the conditioned stimuli (CSs) and the unconditioned stimulus (UCS). An experiment by Perry, Grant, and Schwartz (1977) provides a notable exception to this generalization. Using the grammatical correctness of adjective-noun phrases as the discriminandum, they obtained a large number of "unaware" subjects. These subjects, in contrast to the "aware" subjects, gave very little evidence of conditioned discrimination.

The present experiment employed CS-UCS contingencies of varying degrees of complexity. It was hoped that the contingencies would be sufficiently complex in at least two of the four treatment conditions to produce a substantial number of unaware subjects and, thereby, to make it possible to further explore this issue. Given that those subjects who respond differentially will be almost entirely from the aware subject category, we ask the additional question of whether awareness is a sufficient indicator (in the statistical sense) of performance relative to contingency group.

\section{METHOD}

The present article and two others (Benish \& Grant, 1980a, $1980 \mathrm{~b}$ ) report three aspects of the data from a single study. Only two of the four treatment conditions employed in the entire study are described in Benish and Grant (1980b). The reinforcement contingencies for all four groups during the second stage of the experiment were as follows.
(1) Color group-Reinforcement was signaled by the color of the CS. Half of the subjects in this group received an air puff whenever they saw RED or GREEN displayed in red. Reinforcement for the other half of these subjects was signaled by green stimuli. (2) Word group-Reinforcement was signaled by the verbal component of the CS. Half of the subjects in this group received an air puff whenever they saw RED displayed in either color. Reinforcement for the other half of these subjects was signaled by GREEN. (3) Agree group-Reinforcement was contingent upon the word's being presented in the color it indicated; that is, the CS+ set consisted of RED printed in red letters and GREEN printed in green letters. (4) Clash group-Reinforcement was contingent upon the word's being presented in the inappropriate color; that is, the CS+ set for the clash group was the CS- set for the agree group and vice versa.

Twenty right-handed female and 12 right-handed male subjects were assigned randomly to each of the treatments. Other methodological details can be found in Benish and Grant (1980b).

\section{RESULTS}

Differences in discrimination performance among the contingency conditions during the second stage of the experiment are shown in Figure 1. Percent responding to CS- has been subtracted from percent responding to CS+ and has been plotted against trial block. The upper panel is restricted to data from aware subjects (i.e., subjects who, immediately following the experiment, were able to express a rule that would successfully predict occurrence of the UCS). The data presented in the lower panel of Figure 1 summarize discrimination performance among all subjects.

As can be seen from Figure 1, discrimination was generally much better in the word group, but the superiority of this group disappears when only aware subjects are considered. It follows that the word condition produced a disproportionately large percentage of aware subjects $(80 \%$, compared with $26 \%, 30 \%$, and $40 \%$ for the color, agree, and clash groups, respectively). The hypothesis that awareness was independent of contingency group was rejected by a chi-square test $\left[\chi^{2}(3)=20.77, \mathrm{p}<.001\right]$. Treating awareness as an independent variable, the apparent Awareness by 

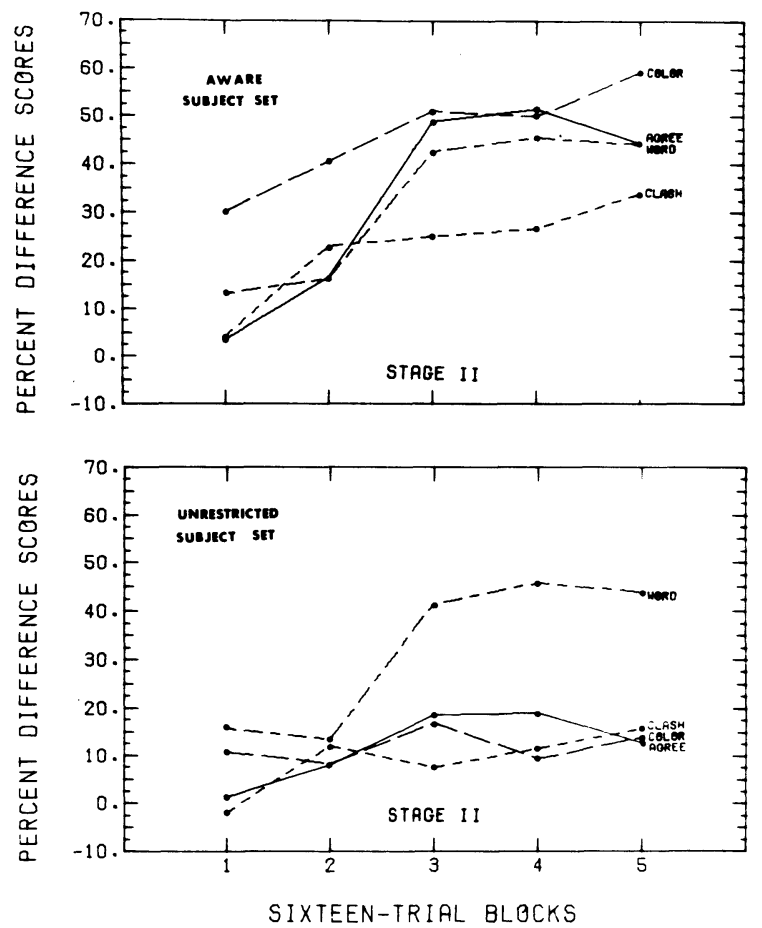

Figure 1. Mean percent difference scores as a function of Stage 2 trial block plotted separately for each group. Data from aware subjects are plotted in the upper panel, and data from all subjects combined are presented in the lower panel.

Reinforcement interaction was highly significant $[F(1,113)=41.53, p<.001]$. Although a significant Reinforcement by Contingency Group effect was found $[F(3,105)=4.16, p<.01]$, none of the contingency group effects was significant at the .10 level when analyses were restricted to data from aware subjects.

A subject was classified as a discriminator if (1) during any trial block he emitted three more conditioned responses (CRs) to $\mathrm{CS}+$ than to $\mathrm{CS}-$, and (2) during each subsequent block he gave at least $50 \%$ more CRs to $\mathrm{CS}+$ than to CS-. This criterion is similar to that adopted by Zajano and Grant (1974). During Stages 1 and 2 of the present experiment, $96 \%$ and $82 \%$, respectively, of the subjects classified as discriminators were also classified as aware. However, only $56 \%$ of the aware subjects during Stage 1 and $62 \%$ of the aware subjects during Stage 2 were found to be discriminators.

No relationship was observed between the V-C classification and the awareness dimension.

\section{DISCUSSION}

The results of this investigation are consistent with the generalization that awareness is a necessary but not a sufficient condition for differential responding in classical eyelid conditioning. Although not every subject identified as a discriminator was also classified as being aware, the proportion was reasonably close to unity (.96 for Stage 1 and .82 for Stage 2), given the limitations of assessing awareness with a brief postexperimental interview or questionnaire (cf. Creelman, 1966, pp. 72-78; Dulany, 1961; Grant, 1973) and the likelihood that some subjects were classified as discriminators as a consequence of random performance fluctuations. On the other hand, given that a subject was aware of the contingencies, the probability that he was also a discriminator was relatively small (.56 for Stage 1 and .62 for Stage 2).

The discrimination task posed by the word condition proved to be quite simple compared with the discriminations required by the other three treatments. Yet, remarkably, no evidence of a word-group superiority remained once attention was restricted to aware subjects. This finding can be accounted for by assuming that complexity of the CS-UCS contingencies affects performance only insofar as it influences an intervening learning state. It can be postulated further that subjects are able to "read out" the contents of this buffer when administered a questionnaire following the experiment.

\section{REFERENCES}

Benish, W. A., \& Grant, D. A. Hemispheric processing in differential classical eyelid conditioning. Bulletin of the Psychonomic Society, 1980, 15, 433-434. (a)

BENISH, W. A., \& GRANT, D. A. Intrastimulus conflict in differential classical eyelid conditioning. Bulletin of the Psychonomic Society, 1980, 15, 428-430. (b)

CREelman, M. B. The experimental investigation of meaning: $A$ review of the literature. New York: Springer, 1966.

Dulany, D. E. Hypotheses and habits in verbal "operant conditioning." Journal of Abnormal and Social Psychology, 1961, 63, 251-264.

Grant, D. A. Cognitive factors in eyelid conditioning. Psychophysiology, 1973, 10, 75-81.

Perry, L. E., Grant, D. A., \& Schwartz, M. Effects of noun imagery and awareness of the discriminative cue upon differential eyelid conditioning to grammatical and ungrammatical phrases. Memory \& Cognition, 1977, 5, 423-429.

Ross, L. E. Cognitive factors in conditioning: The use of masking tasks in eyelid conditioning. In H. H. Kendler \& J. T. Spence (Eds.), Essays in neobehaviorism: A memorial volume to Kenneth $W$. Spence. New York: Appleton-Century-Crofts, 1971.

Zajano, M. J., \& GRant, D. A. Response topography in the acquisition of differential eyelid conditioning. Journal of Experimental Psychology, 1974, 103, 1115-1123.

(Received for publication April 24, 1980.) 